\title{
Factores de prevalencia de mastitis subclínica en vacas lecheras del distrito de Florida, Región Amazonas, Perú
}

\author{
Factors of prevalence of subclinical mastitis in dairy cows in the district of \\ Florida, Amazonas Region, Peru \\ Wigoberto Alvarado C. ${ }^{1}$, Jhony González M. ${ }^{1}$, Carlos Quilcate P. ${ }^{1}$, José Saucedo U. ${ }^{1}$, \\ Joseph Bardales D. ${ }^{1,2}$
}

\section{Resumen}

El objetivo de la investigación fue determinar los principales factores que influyen en la prevalencia de mastitis subclínica en la cuenca lechera de Florida, Región Amazonas, Perú. Se utilizaron 50 vacas de 15 fincas bajo ordeño manual. La prevalencia de mastitis subclínica se determinó mediante el California Mastitis Test (CMT) y el Test DCC (DeLaval Direct Cell Counter). Para determinar los principales factores de prevalencia se utilizó un formato de observación sobre rutinas de ordeño. El análisis de datos se realizó mediante tablas de contingencia, la prueba de chi-cuadrado y correlaciones bivariadas. Se encontró una prevalencia de mastitis subclínica, según el método DCC del 52\% y mediante CMT del 51\%, sin diferencias significativas entre métodos. Los principales factores que influyeron en la prevalencia de mastitis subclínica se debieron a deficientes prácticas de higiene e inadecuadas instalaciones.

Palabras clave: mastitis subclínica; vacas en producción; DCC; CMT; prevalencia; buenas prácticas de ordeño

\section{Abstract}

The aim of this study was to determine the main factors that influence the prevalence of subclinical mastitis in Florida, Amazonas Region, Peru. Fifty cows under hand-milking of 15 farms were used. The prevalence of subclinical mastitis was determined by the California Mastitis Test (CMT) and the DCC Test (DeLaval Direct Cell Counter). Milking

\footnotetext{
${ }^{1}$ Instituto de Investigación en Ganadería y Biotecnología, Universidad Nacional Toribio Rodríguez de Mendoza, Amazonas, Perú

${ }^{2}$ E-mail: joseph.bardales@untrm.edu.pe
}

Recibido: 5 de septiembre de 2018

Aceptado para publicación: 13 de abril de 2019 
routines were observed to determine the main prevalence factors. Data analysis was performed using contingency tables, the chi-square test and bivariate correlations. The prevalence of subclinical mastitis according to the DCC method was $52 \%$ and by CMT was $51 \%$, without significant differences between methods. The main factors that influenced the prevalence of subclinical mastitis were due to poor hygiene practices and inadequate facilities.

Key words: subclinical mastitis; milking cows; DCC; CMT; prevalence; good milking practices

\section{INTRODUCCIÓN}

La crianza de ganado bovino en la región Amazonas, Perú, especialmente en la cuenca lechera de Florida, es la principal actividad económica de las familias campesinas. A pesar de ello, se desarrolla con una débil transferencia de tecnología y la producción es comercializada en mercados informales o derivada a pequeñas plantas artesanales de derivados lácteos, generando una explotación ganadera no competitiva con deficiencias en el manejo sanitario en la preservación de la inocuidad de la leche.

La mastitis ejerce un gran impacto negativo en la producción y la calidad de la leche, traduciéndose en mermas económicas por las pérdidas de producción durante el tratamiento de las vacas enfermas; además de costos por inversión de tiempo y dinero (Bennedsgaard et al., 2003; van Soest et al., 2016). Sin embargo, un gran número de glándulas mamarias afectadas con mastitis no se detecta fácilmente mediante la palpación ni por el examen visual de la leche, de allí que se hace necesario emplear métodos especiales de diagnóstico como el Calfornia Mastitits Test (Schalm y Noorlander, 1957) y el Winsconsin Mastitis Test (Thompson y Postle, 1964) y otros como el contaje de células somáticas (Differential cell count) (DeLaval, 2011).

Los principales agentes que causan la inflamación de la glándula mamaria son Staphylococcus aureus, Escherichia coli,
Corynebacterium bovis y Streptococcus (Godden et al., 2017; Juronen et al., 2018; Heikkilä et al., 2018). Por otro lado, las pérdidas económicas causadas por la mastitis subclínica varían según el patógeno (Gonçalves, 2018), mientras que los factores de riesgo están relacionados a las características del ganadero, al manejo del lote y a las prácticas de ordeño (Curbelo, 2007; Mekonnen, 2017). Por lo tanto, el objetivo de la presente investigación fue determinar la prevalencia y los principales factores de la presencia de mastitis subclínica en la cuenca lechera de Florida, Región Amazonas.

\section{Materiales y Métodos}

\section{Zona de Estudio}

La presente investigación se desarrolló de mayo a noviembre de 2016 en el distrito de Florida, provincia de Bongará, Región Amazonas (Perú). La zona se encuentra ubicada a $2220 \mathrm{msnm}$, presenta una media de precipitación pluvial de $3300 \mathrm{~mm} /$ año y una temperatura media anual de $14{ }^{\circ} \mathrm{C}$, con $87 \%$ de humedad promedio (Salas et al., 2016).

El distrito de Florida cuenta con 7742 cabezas de ganado vacuno y 15000 ha cubiertas con pasturas naturales y cultivadas, donde el 30\% dispone de agua para riego (INEI, 2012). El sistema de ordeño utilizado es manual una vez al día, en la mayoría de los casos con la presencia del ternero. La com- 
posición racial de los hatos es de Simmental, Brown Swiss, sus cruces y animales criollos.

\section{Tamaño Muestral}

El tamaño poblacional estuvo conformado por 2751 vacas en 751 hatos. Para determinar el tamaño muestral se utilizó la fórmula para la afijación óptima de poblaciones finitas ( $p=0.6$ y nivel de confianza del $95 \%$ ), obteniéndose una muestra de 50 vacas en 16 hatos. El muestreo se realizó mediante afijación proporcional a cada estrato (hato), distribuyendo a las vacas por conglomerados según la metodología de Mateu y Casal (2003) para muestreos en epidemiología.

\section{Mastitis Subclínica}

\section{Prueba de Mastitis California (CMT)}

La reacción está relacionada a la cantidad de células somáticas de la leche (RCS). Para la toma de la muestra, se eliminaron los primeros chorros de leche y se tomaron $2 \mathrm{ml}$ de leche de cada cuarto, a los que se adicionó $2 \mathrm{ml}$ de reactivo de CMT (Dodecil sodio sulfato $2 \%$, Cristal violeta $0.0033 \%$ y vehículo acuoso c.s.p. $500 \mathrm{ml}$ ). Se agitó por 10 segundos y los resultados fueron clasificados de acuerdo al método descrito por Pastor y Bedolla (2008) como: (a) Negativa: la mezcla se mantiene liquida ( $<200000$ células/ml); (b) Trazas: ligera viscosidad (200000 500000 células $/ \mathrm{ml}$ ); (c) 1: la mezcla es viscosa, pero no adherida al fondo (400 000 1500000 células $/ \mathrm{ml}$ ); (d) 2: la mezcla es viscosa y adherida al fondo (800 000 - 5000000 células $/ \mathrm{ml}$ ); (e) 3: la mezcla es muy viscosa y fuertemente adherida, formando un solo grumo (>5 000000 células $/ \mathrm{ml}$ ). Para los efectos del estudio, y siguiendo los criterios de NMC (1999), se consideró como mastitis subclínica a las muestras que resultaron con igual o mayor a 200000 células/ml (trazas).

Test DCC (DeLaval Direct Cell Counter)

El RCS se obtuvo con el equipo DeLaval Direct Cell Counter, instrumento portátil, con- tador de células con rango de medición entre 10000 y 4000000 de células somáticas $/ \mathrm{ml}$ (DeLaval, 2011). Se tomó una alícuota de $60 \mu 1$ por medio de un dispositivo comercial (cassette), el cual contiene unos capilares impregnados con una sonda fluorescente marcada con yoduro de propidio. Este dispositivo es colocado en la cámara de lectura del equipo para contabilizar la producción de señales fluorescentes específica para el ADN, siguiendo la metodología propuesta por Berry y Broughan (2007).

\section{Factores de Prevalencia}

Se diseñó una ficha de observación en campo y para entrevista a los productores, siguiendo la metodología propuesta por Santibañez-Ballón et al. (2013), que incluía preguntas relacionadas al animal (raza, edad, número de partos, periodo de lactancia) y prácticas de manejo (lugar de ordeño, inmovilización de patas, uso de maneas limpias, estimulación previa, despunte, amarre de cola, lavado de la ubre, secado con toallas individuales, lavado y secado de manos de los ordeñadores, pre-sellado, tipo de ordeño, número de ordeños al día, ordeño de los casos con mastitis al final, sellado de pezones al finalizar el ordeño, conocimiento sobre prevención de mastitis y número de vacas del hato).

\section{Análisis de Datos}

La información fue analizada mediante estadística descriptiva, tablas de contingencia, prueba de Chi-cuadrado y correlaciones para medir el grado de asociación entre las variables. La prevalencia de mastitis subclínica, según cuartos de la ubre, se determinó mediante tablas de contingencia y el grado de asociación con la raza, edad, periodo de lactancia, número de partos y producción de leche, utilizando la prueba de Chicuadrado $(p<0.05)$. La asociación de las variables prácticas de manejo con la prevalencia de mastitis subclínica se determinó mediante correlaciones bivariadas significativas $(p<0.05)$ y altamente significativas $(p<0.01)$. Se utilizó el software SPSS v. 20 para Windows 7 . 
Cuadro 1. Prevalencia (\%) de mastitis subclínica mediante la prueba de Mastitis California (CMT) y del contaje de células somáticas (DDC) por cuartos de la ubre $(\mathrm{n}=200)$ de vacas en la cuenca lechera de Florida, Amazonas (Perú)

\begin{tabular}{lcccccc}
\hline Método & Reacción & AI & AD & PI & PD & Total (n) \\
\hline CMT & 0 & 80 & 84 & 74 & 74 & 156 \\
& 1 & 10 & 14 & 16 & 16 & 28 \\
& 2 & 2 & 2 & 8 & 8 & 10 \\
& 3 & 8 & & 2 & 2 & 6 \\
\hline DCC & Negativo & 80 & 84 & 74 & 76 & 131 \\
& Positivo & 20 & 16 & 26 & 24 & 69 \\
\cline { 3 - 6 } & & & & & & 200 \\
\hline
\end{tabular}

$\mathrm{Al}$ : anterior izquierdo, AD: anterior derecho, PI: posterior izquierdo, PD: posterior derecho

\section{Resultados y Discusión}

Los promedios de la prevalencia de mastitis subclínica, diagnosticada por CMT $(51 \%)$ y por DCC $(52 \%)$ fueron ligeramente superiores al $48.7 \%$ encontrado por Conde et al. (2016) en Colombia (48.7\%), pero mayores al 38 y $39.5 \%$ reportados en Ecuador y Colombia por De La Cruz González (2011) y Ramírez et al. (2011), respectivamente. Sin embargo, menores al 72.3 y $65.6 \%$, incluyendo o sin incluir trazas, respectivamente, reportado por Santibañez-Ballón et al. (2013) en los andes peruanos, $64 \%$ en Ecuador reportado por Bonifaz y Conlago (2016). Además, Gómez-Quispe et al. (2015) encontraron una prevalencia por cuartos del $48.7 \%$ con sistema de ordeño manual en Apurímac, Perú. Las diferencias entre hallazgos estarían relacionadas a los múltiples factores de riesgos que se encuentran en el manejo, la alimentación y salud de cada hato (Mekonnen et al., 2017).

No se hallaron cuartos ciegos en el estudio. El diagnóstico con CMT muestra que de 200 cuartos muestreados, 44 (22\%) están afectados con algún grado de mastitis, siendo los cuartos posteriores los que presentan mayor presencia de la afección (Cuadro 1).
Bonifaz y Conlago (2016) en Ecuador reportan resultados similares, donde el cuarto más afectado fue el posterior derecho, seguido por el posterior izquierdo; sin embargo, Escobar y Mercado (2008) en Colombia encontró que el anterior derecho fue el cuarto más afectado $(28.9 \%)$

Según el método DCC, hubo 69 cuartos con diagnóstico positivo a mastitis subclínica (RCS $>200000$ células/ml), habiendo mayor positividad en los cuartos posteriores. Al respecto, Sharif y Muhammad (2008) consideran mastitis subclínica un RCS superior a 200000 células/ml. Para disminuir estos niveles de RCS se tiene que mejorar las prácticas de ordeño con enfoques tecnológicos novedosos para que los productores puedan aplicarlos en sus granjas (Nickerson y Oliver, 2014).

Se encontró una menor prevalencia en vacas Simmental, Brown Swiss y Holstein con relación a las cruzadas y criollas $(\mathrm{p}<0.05$; Cuadro 2). Al respecto, Santivañez-Ballón et al. (2013) reportaron que las vacas Holstein tiene una mayor probabilidad de padecer mastitis, mientras que Cuchillo et al. (2010) indican que uno de los factores más relevantes que influyen en los promedios del RCS es la raza del animal. 
Cuadro 2. Prevalencia de mastitis subclínica según raza, etapa de lactancia, número de partos, edad y nivel de producción de leche en la cuenca lechera de Florida, Amazonas (Perú)

\begin{tabular}{llccc}
\hline Variable & Categoría & $\begin{array}{c}\text { Vacas } \\
(\mathrm{n})\end{array}$ & $\begin{array}{c}\text { Mastitis } \\
\text { subclínica } \\
(\%)\end{array}$ & $\begin{array}{c}\text { Chi-cuadrado } \\
\text { (p-valor) }\end{array}$ \\
\hline Raza & Simmental & 22 & $45.5 \pm 0.51^{\mathrm{a}}$ & 0.042 \\
& Brown Swiss & 13 & $53.8 \pm 0.52^{\mathrm{b}}$ & \\
& Holstein & 2 & $50.0 \pm 0.70^{\mathrm{b}}$ & \\
& Cruce & 10 & $60.0 \pm 0.51^{\mathrm{c}}$ & \\
& Criollo & 3 & $66.7 \pm 0.47^{\mathrm{d}}$ & \\
Etapa de lactancia (días) & $1-90$ & 21 & $61.9 \pm 0.50$ & $0.481 \mathrm{NS}$ \\
& $91-150$ & 15 & $46.7 \pm 0.52$ & \\
Número de partos & $151-219$ & 14 & $42.9 \pm 0.52$ & \\
& 1 & 8 & $37.5 \pm 0.52$ & $0.070 \mathrm{NS}$ \\
& 2 & 13 & $61.5 \pm 0.51$ & \\
& 3 & 18 & $55.6 \pm 0.51$ & \\
Edad (años) & $>3$ & 11 & $45.0 \pm 0.52$ & \\
& $2-3$ & 8 & $50.0 \pm 0.53$ & $0.614 \mathrm{NS}$ \\
& $3-4$ & 18 & $61.1 \pm 0.50$ & \\
Nivel de producción & $>4$ & 24 & $45.8 \pm 0.51$ & \\
(1/día) & $1-5$ & 5 & $40.0 \pm 0.54$ & $0.834 \mathrm{NS}$ \\
& $6-10$ & 37 & $54.1 \pm 0.50$ & \\
\hline
\end{tabular}

a,b,c Letras diferentes en la columna de mastitis subclínica indica diferencias significativas $(p<0.05)$. (NS) Sin diferencia significativa $(p>0.05)$

No se hallaron diferencias significativas por efecto de la etapa de lactancia, número de partos, edad o nivel de producción (Cuadro 2). Velásquez y Vega (2012) en la provincia de Huaura-Lima, tampoco encontraron diferencias en prevalencia de mastitis subclínica por efecto del número de parto. No obstante, los casos de mastitis subclínica pueden tornarse crónicos y de esa forma se podría esperar más casos en vacas adultas que en vacas jóvenes (Philpot y Nickerson, 2000).
Hoque et al. (2018) encontraron que una mayor producción de leche se asocia significativamente como factor de riesgo para el inicio de desarrollo de mastitis. Según Hertl et al. (2014), las pérdidas en producción de leche por mastitis varían de acuerdo con el patógeno causante de la infección; sin embargo, Jamali et al. (2018) mencionan que los efectos más importantes de la mastitis en el rendimiento de la producción de leche se dan en casos de mastitis clínica. 
Cuadro 3. Coeficiente de correlación entre la prevalencia de mastitis subclínica con las prácticas de ordeño

\begin{tabular}{lc}
\hline Factores & $\begin{array}{c}\text { Mastitis } \\
\text { subclínica } \\
\text { (coef. de } \\
\text { correlación) }\end{array}$ \\
\hline $\begin{array}{c}\text { El ordeño no se realiza en } \\
\text { un lugar exclusivo }\end{array}$ & $0.826^{* *}$ \\
$\begin{array}{l}\text { Material de piso del } \\
\text { corral de ordeño es de } \\
\text { tierra }\end{array}$ & $0.515^{*}$ \\
$\begin{array}{l}\text { El ordeño no se realiza } \\
\text { bajo techo }\end{array}$ & $0.826^{* *}$ \\
$\begin{array}{l}\text { Realiza despunte antes } \\
\text { del ordeño }\end{array}$ & $-0.541^{*}$ \\
$\begin{array}{l}\text { Realiza lavado adecuado } \\
\text { de ubre }\end{array}$ & $-0.633^{* *}$ \\
$\begin{array}{l}\text { No ordeña al final las } \\
\text { vacas con mastitis }\end{array}$ & $0.826^{* *}$ \\
\hline$* *$ p<0.01; ${ }^{*}$ p $<0.05$ &
\end{tabular}

De la observación directa del proceso de ordeño y encuestas a los productores se halló que el $31.3 \%$ de productores realiza el ordeño en un lugar exclusivo para esta actividad, el $31.3 \%$ lo realizan bajo techo, el $87.5 \%$ realiza la inmovilización de patas, pero solo el $37.5 \%$ usa maneas limpias para esta operación, el $68.8 \%$ de productores realiza una estimulación de pezones previa al ordeño, pero solo el $31.3 \%$ realiza el despunte antes del ordeño, el $75 \%$ amarra la cola de las vacas, el 37.5\% realiza un lavado adecuado de la ubre, pero solo el $12.5 \%$ utiliza toallas individuales para el secado, el $37.5 \%$ de productores se lavan y se secan las manos para ordeñar, solo el $12.5 \%$ realiza el pre-sellado y el $31.2 \%$ ordeña las vacas con mastitis al final de la actividad.

Los principales factores relacionados a la prevalencia de mastitis subclínica en vacas (Cuadro 3) fueron el ordeño que no se lleva a cabo en un lugar exclusivo para esta labor $\left(\mathrm{r}=0.826^{* *}\right)$, material del piso del corral de ordeño $\left(\mathrm{r}=0.515^{*}\right)$ y el ordeño realizado bajo techo $\left(\mathrm{r}=0.826^{* *}\right)$. Por lo tanto, el productor que realiza el ordeño en un lugar con instalaciones adecuadas disminuye la probabilidad de la presencia de mastitis.

El despunte antes del ordeño se correlacionó de manera negativa y significativa con la prevalencia de mastitis subclínica $\left(\mathrm{r}=-0.541^{*}\right)$ (Cuadro 3$)$; es decir que el productor que no realiza el despunte antes del ordeño incrementa la probabilidad de presentar problemas de mastitis en el hato, corroborando los hallazgos de Santman-Berends et al. (2016) quienes mencionan que los factores de riesgo de mastitis pueden ser influenciados por el mismo productor.

En el Cuadro 3 también se observa que el lavado adecuado de la ubre se correlaciona de manera negativa y significativa con la prevalencia de mastitis subclínica $\left(\mathrm{r}=-0.633^{* *}\right)$; es decir que el productor que no realiza un adecuado lavado de ubre incrementa la probabilidad de presentar la enfermedad en el hato. Por último, el ordeño de las vacas con mastitis al final de la actividad se correlaciona de manera positiva y significativa con la prevalencia de mastitis subclínica $\left(\mathrm{r}=0.826^{* *}\right)$; es decir que el productor que no mantiene un orden en el ordeño incrementa la posibilidad de presentar la enfermedad en el hato, coincidiendo con Dodd (1983), quien indica que se requiere llevar un control de los factores de manejo del hato lechero y de las instalaciones para prevenir la mastitis.

\section{Conclusiones}

- La prevalencia de mastitis subclínica en la cuenca lechera de Florida es mayor al $50 \%$, sin diferencias entre métodos de diagnóstico, siendo los cuartos posteriores los más afectados.

- No se halló diferencias de prevalencia según la etapa de lactancia, número de partos, edad y nivel de producción. 
- Los principales factores que influyen en la prevalencia de mastitis subclínica se debieron a deficientes prácticas de higiene e inadecuadas instalaciones para su desarrollo.

\section{Literatura Citada}

1. Bennedsgaard TW, Enevoldsen $C$, Thamsborg SM, Vaarst M. 2003. Effect of mastitis treatment and somatic cell counts on milk yield in Danish organic dairy cows. J Dairy Sci 86: 3174-3183. doi: 10.3168/jds.S0022-0302(03)73920-4

2. Berry E, Broughan J. 2007. Use of the DeLaval cell counter (DCC) on goats' milk. J Dairy Res 74: 345-348. doi: 10.1017/S0022029907002592

3. Bonifaz N, Conlago F. 2016. Prevalencia e incidencia de mastitis bovina mediante la prueba de California Mastitis Test con identificación del agente etiológico, en Paquiestancia, Ecuador. La Granja 24: 43-52. doi: 10.17163/ lgr.n24.2016.04

4. Conde LAA, Jaramillo WFO, Hoyos NP. 2016. Concordancia entre dos pruebas diagnósticas de mastitis subclínica en la hacienda Londoburgo-Pereira Risaralda Colombia. Tesis de Médico Veterinario y Zootecnista. Risaralda, Colombia: Univ. Tecnológica de Pereira. 55 p.

5. Cuchillo Z, Dauqui V, Campos G. 2010. Factores que inciden en el recuento de células somáticas (RCS) y calidad de la leche. Tesis de Ing. Zootecnista. Palmira, Colombia: Univ. Nacional de Colombia, sede Palmira. $58 \mathrm{p}$.

6. Curbelo RJE. 2007. Relación entre los recuentos de células somáticas, prácticas de manejo y patógenos causantes de mastitis en hatos lecheros de Puerto Rico. Tesis de Maestría. Mayagüez, Puerto Rico: Univ. de Puerto Rico Recinto de Mayagüez. 89 p.
7. De la Cruz González EG. 2012. Correlación de los métodos California Mastitis Test (CMT), conductividad eléctrica (CE) y conteo de células somáticas (CCS) en el laboratorio de calidad de leche de la UPS. Tesis de Ing. Agropecuario. Cayambe, Ecuador: Univ. Politécnica Salesiana. 42 p.

8. Dodd FH. 1983. Mastitis-progress on control. J Dairy Sci 66: 1773-1780. doi: 10.3168/jds.S0022-0302(83)82005-0

9. DeLaval. 2011. Manual de instrucciones. Madrid. [Internet]. Disponible en: https://www.somatco.com/DeLavalcell-counter-DCC.pdf

10. Escobar ER, Mercado CD. 2008. Determinación de mastitis subclínica mediante la prueba mastitis california test (CMT) y la correlación del periodo de lactancia del animal con los cuartos mamarios afectados en bovinos (Bos indicus y cruces) de empresas ganaderas en el municipio de Since-Sucre. Tesis de Ing. Zootecnista. Sincelejo, Colombia: Univ. de Sucre. 87 p.

11. Godden SM, Royster E, Timmerman J, Rapnicki P, Green H. 2017. Evaluation of an automated milk leukocyte differential test and the California Mastitis Test for detecting intramammary infection in early-and late-lactation quarters and cows. J Dairy Sci 100: 6527-6544. doi: 10.3168/ jds.2017-12548

12. Gómez-Quispe OE, SantivañezBallón CS, Arauco-Villar F, EspezuaFlores OH, Manrique-Meza J. 2015. Criterios de interpretación para California Mastitis Test en el diagnóstico de mastitis subclínica en bovinos. Rev Inv Vet Perú 26: 86-95. doi: 10.15381/ rivep.v26i1.10912

13. Gonçalves JL, Kamphuis C, Martins CMMR, Barreiro JR, Tomazi T, Gameiro A, Hogeveen H, et al. 2018. Bovine subclinical mastitis reduces milk yield and economic return. Livest Sci 210: 25-32. doi: 10.1016/j.livsci.2018.01.016 
14. Heikkilä AM, Liski E, Pyörälä S, Taponen S. 2018. Pathogen-specific production losses in bovine mastitis. $\mathrm{J}$ Dairy Sci 101:9493-9504. doi: 10.3168/ jds.2018-14824

15. Hertl JA, Schukken YH, Welcome FL, Tauer L W, Gröhn YT. 2014. Pathogen-specific effects on milk yield in repeated clinical mastitis episodes in Holstein dairy cows. J Dairy Sci 97: 1465-1480. doi: 10.3168/jds.2013-7266

16. Hoque MN, Das ZC, Rahman ANMA, Haider MG, Islam MA. 2018. Molecular characterization of Staphylococcus aureus strains in bovine mastitis milk in Bangladesh. Int J Vet Sci Med 6: 53-60. doi: 10.1016/j.ijvsm.2018.03.008

17. [INEI] Instituto Nacional de Estadística e Informática. 2012. IV Censo Nacional Agropecuario. Perú. [Internet]. Disponible en: http://censos.inei.gob.pe/ cenagro/tabulados/

18. Jamali H, Barkema HW, Jacques M, Lavallée-Bourget EM, Malouin F, Saini V, Stryhn H, et al. 2018. Invited review: incidence, risk factors, and effects of clinical mastitis recurrence in dairy cows. J Dairy Sci 101: 4729-4746. doi: 10.3168/jds.2017-13730

19. Juronen D, Kuusk A, Kivirand K, Rinken A, Rinken T. 2018. Immunosensing system for rapid multiplex detection of mastitis-causing pathogens in milk. Talanta 178: 949-954. doi: 10.1016/j.talanta.2017.10.043

20. Mateu E, Casal J. 2003. Tamaño de la muestra. Rev Epidemiol MedPrev 1:8-14.

21. Mekonnen SA, Koop G, Melkie ST, Getahun CD, Hogeveen H, Lam TJ. 2017. Prevalence of subclinical mastitis and associated risk factors at cow and herd level in dairy farms in North-West Ethiopia. Prev Vet Med 145: 23-31. doi: 10.1016/j.prevetmed.2017.06.009

22. [NMC] National Mastitis Council. 1999. Laboratory handbook on bovine mastitis (Revised Ed), Madison, WI: NMC. 222 p.
23. Nickerson SC, Oliver SP. 2014. How well have United States dairy producers adopted mastitis-control technologies for reducing herd somatic cell counts improving milk quality? The Professional Animal Scientist 30: 115-124. doi: 10.15232/S1080-7446(15)30098-X

24. Pastor JI, Bedolla JLC. 2008. Determinación de la prevalencia de mastitis bovina en el municipio de Tarímbaro, Michoacán, mediante la prueba de California. REDVET 9(10). [Internet]. Disponible en: http://www.vete-rinaria.org/revistas/redvet/n101008/101004.pdf

25. Philpot W, Nickerson S. 2000. Ganando la lucha contra la mastitis. Naperville, IL, USA: Wesfalia Surge. 192 p.

26. Ramírez N, Arroyave O, CerónMuñoz M, Jaramillo M, Cerón J, Palacio LG. 2011. Factores asociados a mastitis en vacas de la microcuenca lechera del altiplano norte de Antioquia, Colombia. Rev Med Vet 22: 31-42. doi: 10.19052/mv.562

27. Salas R, Castillo EB, Oliva M. 2016. Dinámica multitemporal de índices de deforestación en el distrito de Florida, departamento de Amazonas, Perú. INDES 2: 18-27. doi: 10.25127/indes.20142.59

28. Santivañez-Ballón C, Gómez-Quispe OE, Cárdenas-Villanueva L, Escobedo-Enríquez M, Bustinza Cardenas R, Sanchez J. 2013. Prevalencia y factores asociados a la mastitis subclínica bovina en los Andes peruanos. Vet Zootec 7: 92-104. doi: 10.17151/ vetzo.2013.7.2.7

29. Schalm O, Noorlander D. 1957. Experiments and observations leading to the development of California mastitis test. J Am Vet Med Assoc 130: 199-204.

30. Santman-Berends IMGA, Swinkels JM, Lam TJGM, Keurentjes J, van Schaik G. 2016. Evaluation of udder health parameters and risk factors for clinical mastitis in Dutch dairy herds in the context of a restricted antimicrobial usage policy. J Dairy Sci 99: 2930-2939. doi: $10.3168 /$ jds.2015-10398 
31. Sharif A, Muhammad G 2008. Somatic cell count as an indicator of udder health status under modern dairy production: a review. Pak Vet J 28: 194-200.

32. Thompson DI, Postle DS. 1964. The Wisconsin Mastitis Test - an indirect estimation of leucocytes in milk. J Milk Food Technol 27: 271.
33. van Soest FJ, Santman-Berends IM, Lam TJ, Hogeveen H. 2016. Failure and preventive costs of mastitis on Dutch dairy farms. J Dairy Sci 99: 8365-8374. doi: 10.3168/jds.2015-10561

34. Velásquez, C, Vega J. 2012. Calidad de la leche y mastitis subclínica en establos de la provincia de Huaura, Lima. Rev Inv Vet Perú 23: 65-71. doi: 10.15381/ rivep.v23i1.883 